\title{
Awesome allotropy
}

\section{The discovery of $\mathrm{C}_{60}$ - a molecular allotrope of carbon - marked a pivotal moment in the field of nanotechnology. Twenty five years on, carbon remains the element of choice for simple but functional materials.}

When an element exists in more than one structural form, it is said to exhibit allotropy and the forms are called allotropes. This definition, drilled into most of us during our high-school years, is usually exemplified by the most common, naturally occurring allotropes of carbon, namely diamond and graphite. However, 25 years ago this month, the world of carbon allotropes was dramatically altered with the publication in Nature of the discovery of a new molecular allotrope of carbon $\mathrm{C}_{60}$, or buckminsterfullerene ${ }^{1}$. Not only did this discovery reveal an experimental demonstration of a long-standing theoretical challenge and offer an exciting and versatile molecule for chemists and physicists to probe, but it also reflecting the advances in nanotechnology over the past 25 years - meant that $\mathrm{C}_{60}$ had become paramount in our way of thinking of molecules and materials at the nanoscale.

The story behind the Nature publication begins with a team of scientists -

Harry Kroto, Jim Heath, Sean O’Brien, Robert Curl and Richard Smalley investigating the chemistry of carbon clusters present in the interstellar medium. They were particularly interested in small clusters; from their first experiment, however, they saw peaks in their mass spectra corresponding to larger clusters with even numbers of carbon atoms. Under certain experimental conditions, the peak corresponding to 60 carbon atoms was found to be hugely dominant, and hence the most stable cluster. The second most prominent peak corresponded to 70 carbon atoms and, together, these signals representative of $\mathrm{C}_{60}$ and $\mathrm{C}_{70}$ - were noted in subsequent experiments, as Jim Heath, the graduate student of Richard Smalley, recounts in our interview ${ }^{2}$. Although the team of scientists was not the first to see such a distribution of carbon clusters ${ }^{3}$, it was they who appreciated the significance of their findings and, by recognizing that the amazing stability of 60 carbon atoms must result in a closed-up arrangement, were able, over a period of only two weeks, to come up with a rational structure for $\mathrm{C}_{60}$.

Nowadays, the existence and structure of $\mathrm{C}_{60}$ are so well accepted, and fullerenes have been so exhaustively studied, that it is difficult to imagine a different world in which controversy reigned: for several years after the discovery there were many scientists who doubted whether the structure was correct $^{4}$. For example, a research group based at

Exxon who had a history of working with carbon clusters before the 1985 discovery $^{3}$, published an article $e^{5}$ in the Journal of the American Chemical Society, entitled ' $\mathrm{C}_{60} \mathrm{La}$ : a deflated soccer ball?'. In this article they proposed that a lanthanum atom was attached to the edge of graphite flakes rather than encased within spherical $\mathrm{C}_{60}$ (ref. 6). The problem was that only about $1,000 \mathrm{C}_{60}$ molecules could be isolated at one time, and it wasn't possible to image or use spectroscopic techniques to prove the structure. So, until the large-scale synthesis and $\mathrm{X}$-ray diffraction analysis of $\mathrm{C}_{60}$ were reported by Huffman and Krätschmer ${ }^{7}$ the 'doubters' remained vocal and few advances were made in the field. Nonetheless, for the team behind the discovery, their structural hypothesis of $\mathrm{C}_{60}$ explained perfectly all that they had witnessed experimentally. Time proved them right, and Kroto, Smalley and Curl were awarded the Nobel Prize in Chemistry in 1996.

$\mathrm{C}_{60}$, and fullerenes more generally, were just the beginning of a new era of carbon allotropes. In his Commentary, Andreas Hirsch discusses the evolution of synthetic carbon allotropes from fullerenes, to carbon nanotubes and graphene - including their syntheses, properties and applications ${ }^{8}$. In particular, the unique and unprecedented electronic, magnetic and materials properties of the nanoscale allotropes, and hence their incorporation into devices - such as the use of fullerenes in organic solar cells - has made them materials of broad interest. The potential that graphene may have on future technology in several fields is recognized in the 2010 Nobel Prize in Physics, awarded to Andre Geim and Konstantin Novoselov for their work in isolating graphene from graphite and in unveiling its extraordinary electronic properties ${ }^{9}$.
Also in this issue and sticking with the theme of carbon, but on a more global scale, James Tour, Carter Kittrell and Vicki Colvin discuss the role of carbon-based natural resources in future sustainable energy initiatives ${ }^{10}$. Labelled as 'green carbon', this concept - creating efficient and strategic ways to use carbon-based resources, while minimizing the environmental and economic impact - should aid in the transition from the traditional burning of fossil fuels to renewable energy resources.

In his Nobel lecture, Smalley said "We are still in the process of discovering all the other consequences of the genius that is wired into carbon atoms". Although the future is hard to predict in science, in the case of synthetic forms of carbon for which properties and applications are a potential gold mine, the discovery of new materials by wiring carbon atoms together in different ways is a tantalizing prospect. There is now much current research focused on the synthesis of carbon forms, for example, yne-diamond ${ }^{11}$ and structurally well-defined molecular toroidal carbon nanotubes ${ }^{12}$, which have only previously been speculated on from a theoretical standpoint.

Looking back over the past 25 years, although $\mathrm{C}_{60}$ itself has not made our everyday lives immeasurably better, its discovery is an event that marked the beginning of a change of attitude in science towards the synthesis and applications of nanoscale materials. As a consequence of its discovery, $\mathrm{C}_{60}$ has indirectly and profoundly impacted our lives, and will continue to do so.

\section{References}

1. Kroto, H., Heath, J. R., O'Brien, S. C., Curl, R. F. \& Smalley, R. E. Nature 318, 162-163 (1985)

2. Nature Mater. 9, 876-877 (2010).

3. Rohlfing, E. A., Cox, D. M. \& Kaldor, A. J. Chem. Phys. 81, 3322-3330 (1984)

4. Toumey, C. Nature Nanotech. 5, 693-694 (2010).

5. Cox, D. M., Trevor, D. J., Reichmann, K. C. \& Kaldor, A. J. Am. Chem. Soc. 108, 2457-2458 (1986).

6. Heath, J. R. et al. J. Am. Chem. Soc. 107, 7779-7780 (1985).

7. Krätschmer, W., Lamb, L. D., Fostiropoulos, K. \& Huffman, D. R. Nature 347, 354-358 (1990).

8. Hirsch, A. Nature Mater. 9, 868-871 (2010).

9. Novoselov, K. S. et al. Science 306, 666-669 (2004).

10. Tour, J. M., Kittrell, C. \& Colvin, V. L. Nature Mater. 9, 871-874 (2010).

11. Itzhaki, L., Altus, E., Basch, H. \& Hoz, S. Angew. Chem. Int. Ed. 44, 7432-7435 (2005)

12. Chuang, C. Fan, Y.-C. \& Jin, B.-Y. J. Chem. Inf. Model. 49, 361-368 (2009). 